\title{
SURFACE WAVE RUNNING ALONG THE EDGE OF AN ELASTIC WEDGE
}

\author{
I. V. KAMOTSKII
}

\begin{abstract}
The existence of the waves mentioned in the title is proved for the case of an acute wedge.
\end{abstract}

\section{§1. INTRODUCTION}

For the first time, the existence of surface waves in elastic wedges was conjectured in the papers [1, 2] on the basis of results of numerical calculations. It was discovered that such waves oscillate along the edge of the wedge, decaying exponentially far from the edge, and that the velocity of their propagation depends on the wedge's angle and can be considerably less than that of the Rayleigh waves. This latter fact opens a way to use such waves in lag lines.

After the surface waves had been discovered, the corresponding studies developed in several directions; see, e.g., [3, 4. However, the proof that surface waves do exist remained an open question. In the present paper we prove the existence of such waves in acute wedges. Our method is based on variational considerations. Namely, we show that the frequencies for which surface waves may arise correspond to the eigenvalues of a certain selfadjoint operator. Next, in $\S 3$, we use the variational principle to prove the existence of eigenvalues located off the continuous spectrum. This way of argumentation is traditional; see, e.g., [5-7], where it was used for the study of eigenfunctions in waveguide-like domains.

The author is deeply grateful to V. M. Babich for setting the problem and for his permanent attention, and to M. Sh. Birman for valuable remarks.

\section{§2. Setting of the Problem}

Let $\left.\Omega=\left\{x_{1}, x_{2}\right): r>0, \phi \in(0, \Phi)\right\}$ be an angle on the plane $\mathbb{R}^{2}$; here $(r, \phi)$ are polar coordinates. We consider the problem of steady-state oscillations of an isotropic elastic wedge $K=\Omega \times \mathbb{R}$ with free boundary:

$$
\begin{aligned}
-\partial_{m} \sigma_{n m} & =\omega^{2} U_{n} \quad \text { in } K, \\
\sigma_{n m} \nu_{m} & =0 \text { on } \partial K,
\end{aligned}
$$

where $n, m=1,2,3, \sigma_{n m}=\sigma_{n m}\left(\partial_{1}, \partial_{2}, \partial_{3} ; U\right)=\lambda \partial_{l} U_{l} \delta_{n m}+\mu\left(\partial_{m} U_{n}+\partial_{n} U_{m}\right)$ are the components of the stress tensor, $\lambda$ and $\mu$ are the Lamé coefficients, $\delta_{n m}$ is the Kronecker symbol, $U=\left(U_{1}, U_{2}, U_{3}\right)$ is the vector of displacements, and $\left(\nu_{1}, \nu_{2}, \nu_{3}\right)$ is the outward normal vector. The operators on the left-hand sides in (2.1) and (2.2) will be denoted

2000 Mathematics Subject Classification. Primary 74J15.

Key words and phrases. Surface wave, Reyleigh wave, acute wedges, variational principle.

Supported by RFBR, grant no. 07-01-00548. 
by $L\left(\partial_{1}, \partial_{2}, \partial_{3}\right)$ and $N\left(\partial_{1}, \partial_{2}, \partial_{3}\right)$, respectively. Then the Betti formula, valid for any smooth vector-valued function $U=\left(U_{1}, U_{2}, U_{3}\right)$ with bounded support, takes the form

$$
(L U, U)_{K}+(N U, U)_{\partial K}=a_{K}(U, U)
$$

where $a_{K}(U, U)=\int_{K} a\left(\partial_{1}, \partial_{2}, \partial_{3} ; U, U\right) d x$ and $a\left(\partial_{1}, \partial_{2}, \partial_{3} ; U, U\right)=\sigma_{n m} \overline{\partial_{m} U_{n}}$. The quadratic form $a_{K}$ coincides with twice the elastic energy functional.

A solution of problem (2.1), (2.2) is sought in the form

$$
U\left(x_{1}, x_{2}, x_{3}\right)=u\left(x_{1}, x_{2}\right) \exp \left(i k x_{3}\right),
$$

where $k$ is the wave number. Plugging (2.4) in (2.1), (2.2) and separating the oscillating factor, we obtain the problem

$$
\begin{aligned}
L(i k) u & =\omega^{2} u \text { in } \Omega, \\
N(i K) u & =0 \text { on } \partial \Omega .
\end{aligned}
$$

Here $L(i k)=L\left(\partial_{1}, \partial_{2}, i k\right), N(i k)=N\left(\partial_{1}, \partial_{2}, i k\right)$. We have a formula of integration by parts, similar to $(2.3)$ :

$$
(L(i k) u, u)_{\Omega}+(N(i k) u, u)_{\partial \Omega}=a_{\Omega}(i k ; u, u), \quad u \in H^{2}(\Omega),
$$

as well as an analog of Korn's inequality (see [8]),

$$
\left\|u ; H^{1}(\Omega)\right\|^{2} \leq C\left(a_{\Omega}(i k ; u, u)+\left\|u ; L_{2}(\Omega)\right\|^{2}\right),
$$

for $u \in H^{1}(\Omega)$. Here

$$
a_{\Omega}(i k ; u, u)=\int_{\Omega} a\left(\partial_{1}, \partial_{2}, i k ; u, u\right) d x_{1} d x_{2} .
$$

We note that the space of functions for which the right-hand side of (2.8) is finite coincides with $H^{1}(\Omega)$; see $[8$ for the case of smooth domains and 9 for Lipschitz domains. The positive form (2.9) is closed on $d[a(i k)]=H^{1}(\Omega)$; therefore, it determines a unique selfadjoint operator $A(i k)$ with domain $\mathcal{D}(i k)) \subset d[a(i k)]$. On the other hand, the operator $A(i k)$ can be defined as the Friedrichs extension of a symmetric operator $\widehat{A}(i k)$; specifically, $\widehat{A}(i k)$ is defined on the $H^{2}(\Omega)$-functions that satisfy the boundary condition (2.6) and acts by the rule $\hat{A}(i k) u=L(i k) u$.

Thus, the existence problem for surface waves reduces to finding the point spectrum of the selfadjoint operator $A(i k)$.

\section{§3. Spectrum of the Problem operator}

Theorem 2.1. The interval $\left(-\infty, c_{R}^{2} k^{2}\right)$, where $c_{R}$ denotes the velocity of the Rayleigh wave, contains no points of the essential spectrum of the operator $A(i k)$.

The proof will be split into several lemmas.

Lemma 2.2. We have

$$
a_{\mathbb{R}_{+}^{2}}(i k ; u, u) \geq c_{R}^{2} k^{2}\left\|u ; L_{2}\left(\mathbb{R}_{+}^{2}\right)\right\|^{2}, \quad u \in H^{1}\left(\mathbb{R}_{+}^{2}\right),
$$

where $\mathbb{R}_{+}^{2}=\left\{\left(x_{1}, x_{2}\right): x_{2}>0\right\}$.

The proof employs the Fourier transformation and the properties of the Rayleigh wave and can be found in [6.

Lemma 2.3. For any $\varepsilon>0$, we have

$$
a_{\Omega}(i k ; u, u) \geq\left(c_{R}^{2} k^{2}-\varepsilon^{2}\right)\left\|u ; L_{2}(\Omega)\right\|^{2}-C\left\|u ; L_{2}\left(\Omega \cap\left\{r<\varepsilon^{-1}\right\}\right)\right\|^{2},
$$

where $u \in H^{1}(\Omega)$ and $C$ is a constant independent of $u$ and $\varepsilon$. 
Proof. Let $\chi_{1}$ and $\chi_{2}$ be two smooth cut-off functions such that $\chi_{1}(r)=0$ for $r<2$, $\chi_{2}(r)=0$ for $r<1$, and

$$
\chi_{1}^{2}+\chi_{2}^{2} \equiv 1
$$

We multiply $a_{\Omega}(i k ; u, u)$ by $(3.2)$, bring the cut-off functions under the differentiation sign, and use (3.2), obtaining

$$
a_{\Omega}(i k ; u, u)=\sum_{j=1}^{2}\left(a_{\Omega}\left(i k ; \chi_{j} u, \chi_{j} u\right)-\int_{\Omega} a\left(\nabla_{\chi_{j}}, 0 ; u, u\right) d x_{1} d x_{2}\right) .
$$

Using the location of the supports of our cut-off functions and the fact that the second term in parentheses in (3.3) does not involve differentiation, we obtain

$$
a_{\Omega}(i k ; u, u) \geq a_{\Omega}\left(i k ; \chi_{2} u, \chi_{2} u\right)-C_{1}\left\|u ; L_{2}(\Omega \cap\{r<2\})\right\|^{2} .
$$

For brevity, we denote $v=\chi_{2} u$. In order to apply Lemma 2.2, we use an "angle partition of unity", namely, we put $\rho_{j}(x)=\chi_{j}(3 \phi / \Phi)$. As above, we find

$$
a_{\Omega}(i k ; v, v)=\sum_{j=1}^{2}\left(a_{\Omega}\left(i k ; \rho_{j} v, \rho_{j} v\right)-\int_{\Omega} a\left(\nabla \rho_{j}, 0 ; v, v\right) d x_{1} d x_{2}\right) .
$$

The functions $\rho_{1} v$ and $\rho_{2} v$ can be extended by zero to the upper half-plane, and we can apply Lemma 2.2 to these extensions. Recalling (3.2), from (3.5) we deduce the inequality

$$
a_{\Omega}(i k ; v, v) \geq c_{r}^{2} k^{2}\left\|v ; L_{2}(\Omega)\right\|^{2}-\sum_{j=1}^{2} \int_{\Omega} a\left(\nabla \rho_{j}, 0 ; v, v\right) d x_{1} d x_{2}
$$

Since the functions $\rho_{j}$ depend on the angle variable only, the absolute values of their derivatives, occurring in the subtrahend in (3.6), do not exceed $\mathrm{Cr}^{-1}$. Therefore,

$$
\begin{aligned}
a_{\Omega}(i k ; v, v) & \geq c_{R}^{2} k^{2}\left\|v ; L_{2}(\Omega)\right\|^{2}-C_{2}\left\|r^{-1} v ; L_{2}(\Omega)\right\|^{2} \\
& \geq\left(c_{R}^{2} k^{2}-\varepsilon^{2}\right)\left\|v ; L_{2}(\Omega)\right\|^{2}-C_{2}\left\|v ; L_{2}\left(\Omega \cap\left\{r<\varepsilon^{-1}\right\}\right)\right\|^{2} .
\end{aligned}
$$

Now, the statement of the lemma follows from estimates (3.4) and (3.7).

We return to the proof of Theorem 2.1. We argue as in [10]. Suppose that a point $\omega_{1} \in\left(-\infty, c_{R}^{2} k^{2}\right)$ belongs to the essential spectrum of the operator $A(i k)$. Then there exists an orthonormal sequence $u_{n} \in D(A(i k))$ such that $\left(A(i k)-\omega_{1}\right) u_{n} \rightarrow 0$ (see [11]). Consequently,

$$
a_{\Omega}\left(i k ; u_{n}, u_{n}\right) \rightarrow \omega_{1}, \quad n \rightarrow+\infty .
$$

On the other hand, choosing $\varepsilon=\left(c_{R}^{2} k^{2}-\omega_{1}\right)^{1 / 2} / 4$ in Lemma 2.3 , we obtain

$$
a_{\Omega}\left(i k ; u_{n}, u_{n}\right)+C\left\|u_{n} ; L_{2}\left(\Omega \cap\left\{r<\varepsilon^{-1}\right\}\right)\right\|^{2} \geq\left(c_{R}^{2} k^{2}+\omega_{1}\right) / 2 .
$$

The sequence $u_{n}$ is orthonormal, and, by (3.8) and (2.8), the quantities $\left\|u_{n} ; H^{1}(\Omega)\right\|$ are bounded. Therefore, we can find a subsequence $u_{n_{p}}$ tending to zero in $L_{2}\left(\Omega \cap\left\{r<\varepsilon^{-1}\right\}\right)$. Thus, we have

$$
a_{\Omega}\left(i k ; u_{n_{p}}, u_{n_{p}}\right)+C\left\|u_{n_{p}} ; L_{2}\left(\Omega \cap\left\{r<\varepsilon^{-1}\right\}\right)\right\|^{2} \rightarrow \omega_{1}, \quad n \rightarrow+\infty,
$$

which contradicts (3.9) because $c_{R}^{2} k^{2}>\omega_{1}$.

Remark 1. Theorem 2.1 states that the essential spectrum of $A(i k)$ is contained in the interval $\left[c_{R}^{2} k^{2},+\infty\right)$. However, it is not difficult to construct an appropriate singular sequence showing that actually the two sets mentioned above coincide.

Theorem 2.4. If $\Phi<\pi / 2$, then the interval $\left(0, c_{R}^{2} k^{2}\right)$ contains a point of the point spectrum of the operator $A(i k)$. 
I. V. KAMOTSKII

Proof. By the variational principle (see [1]]), the lower bound of the selfadjoint operator $A(i k)$ can be found by the formula

$$
\begin{aligned}
\underline{\sigma}(A(i k)) & =\inf \Psi(u), \quad u \in d[A(i k)], \quad u \neq 0, \\
\Psi(u) & =\frac{a_{\Omega}(i k ; u, u)}{(u, u)} .
\end{aligned}
$$

By Theorem 2.1, it suffices to show that there is $u^{\text {test }} \in d(i k)$ such that

$$
\Psi\left(u^{\text {test }}\right)<c_{R}^{2} k^{2} .
$$

Let $U^{R}\left(x_{1}, x_{2}, x_{3}\right)$ be a Rayleigh wave that propagates in the direction of $(0,0,1)$ and leaves the plane $x_{2}=0$ free of stresses. Such a wave is representable in the form

$$
U^{R}\left(x_{1}, x_{2}, x_{3}\right)=\exp \left(i k x_{3}\right) u^{R}\left(x_{2}\right),
$$

and the exponentially decaying vector-valued function $u^{R}\left(x_{2}\right)$ delivers the minimum, equal to $c_{R}^{2} k^{2}$, to the functional

$$
\Psi_{1}(u)=\int_{0}^{\infty} a\left(i k ; u^{R}, u^{R}\right) d x_{2}\left(\int_{0}^{\infty} u^{R} \overline{u^{R}} d x_{2}\right)^{-1} .
$$

This yields the estimate

$$
\int_{0}^{N} a\left(i k ; u^{R}, u^{R}\right) d x_{2}<c_{R}^{2} k^{2} \int_{0}^{N} u^{R} \overline{u^{R}} d x_{2},
$$

for any $N>0$. Indeed, otherwise there must exist a function of the form (3.12) that represents a surface wave leaving the plane $x_{2}=N$ free of stresses and propagating with velocity less than that of the Rayleigh wave.

We define a test sequence by the formula

$$
u_{n}^{\text {test }}\left(x_{1}, x_{2}\right)=\exp \left(-n^{-1} x_{1}\right) u_{R}\left(x_{2}\right), \quad n=1,2, \ldots .
$$

Plugging this in (3.10) and using (3.13) and the fact that $u_{R}\left(x_{2}\right)$ decays exponentially as $x_{2} \rightarrow+\infty$, we obtain

$$
\begin{aligned}
\Psi & \left(u_{n}^{\text {test }}\right)=\frac{a_{\Omega}\left(i k ; u_{n}^{\text {test }}, u_{n}^{\text {test }}\right)}{\left(u_{n}^{\text {test }}, u_{n}^{\text {test }}\right)} \\
= & \frac{\int_{0}^{+\infty} e^{-2 n^{-1} x_{1}} \int_{0}^{\text {tan } \Phi x_{1}}\left(a\left(i k ; u^{R}, u^{R}\right)+\mu n^{-2} u^{R} \overline{u^{R}}\right) d x_{2} d x_{1}}{\left(u_{n}^{\text {test }}, u_{n}^{\text {test }}\right)} \\
= & \frac{\int_{0}^{+\infty} e^{-2 n^{-1} x_{1}} \int_{0}^{\tan \Phi x_{1}} a\left(i k ; u^{R}, u^{R}\right) d x_{2} d x_{1}}{\left(u_{n}^{\text {test }}, u_{n}^{\text {test }}\right)}+\mu n^{-2} \\
= & c_{R}^{2} k^{2}+\frac{\int_{0}^{+\infty} e^{-2 n^{-1} x_{1}}\left(c_{R}^{2} k^{2} \int_{0}^{\tan \Phi x_{1}} u^{R} \overline{u^{R}} d x_{2}-\int_{0}^{\tan \Phi x_{1}} a\left(i k ; u^{R}, u^{R}\right) d x_{2}\right) d x_{1}}{\left(u_{n}^{\text {test }}, u_{n}^{\text {test }}\right)} \\
& +\mu n^{-2}<c_{R}^{2} k^{2}-C n^{-1}+C_{1} n^{-2},
\end{aligned}
$$

where $C$ and $C_{1}$ are positive constants independent of $n$. Putting $u^{\text {test }}=u_{n}^{\text {test }}$ for $n$ sufficiently large, we see that inequality (3.11) is fulfilled.

Remark 2. The above proof does not cover the case of the right angle (the constant $C$ vanishes). Nevertheless, Theorem 2.4 can be proved also in this situation, under the additional requirement that $\lambda \neq 0$, with the help of arguments similar to those in [7. 
Theorem 2.1 ensures the solvability of problem (2.5), (2.6) in the class $H^{1}(\Omega)$. Moreover, arguing as in [10], it is not hard to show that the solution decays exponentially. Namely, there exists a constant $c>0$ depending on $\lambda$ and $\mu$ and such that if $u$ is the solution of $(2.5),(2.6)$ and $\omega^{2} \in\left(0, c_{R}^{2} k^{2}\right)$, then

$$
\exp \left\{c\left(c_{R}^{2} k^{2}-\omega^{2}-\varepsilon\right)^{1 / 2}\left(x_{1}^{2}+x_{2}^{2}\right)^{\frac{1}{2}}\right\} u \in H^{1}(\Omega)
$$

for an arbitrary $\varepsilon \in\left(0, c_{R}^{2} k^{2}-\omega^{2}\right)$.

\section{REFERENCES}

[1] A. A. Maradudin et al., Vibrational edge modes in finite crystals, Phys. Rev. B 6 (1972), 1106-1111.

[2] P. E. Lagasse, Analysis of a dispersion-free guides for elastic waves, Electron. Lett. 8 (1972), no. 15, 372-373.

[3] V. V. Krylov, Geometro-acoustical approach to description of localized modes for vibration of a solid elastic wedge, Zh. Tekhn. Fiz. 60 (1990), no. 2, 1-7. (Russian)

[4] A. V. Shanin, Excitation and scattering of a wedge wave in an elastic wedge with angle close to $180^{\circ}$, Akust. Zh. 43 (1997), no. 3, 402-408. (Russian)

[5] D. V. Evans, M. Levitin, and D. Vassiliev, Existence theorems for trapped modes, J. Fluid Mech. 261 (1994), 21-31. MR1265871 (94m:76113)

[6] A. S. Bonnet-Ben Dhia, J. Duterte, and P. Joly, Mathematical analysis of elastic surface waves in topographic waveguides, Math. Models Methods Appl. Sci. 9 (1999), 755-798. MR1697393 (2000d:74036)

[7] I. V. Kamotskiŭ and S. A. Nazarov, Elastic waves localized near periodic families of defects, Dokl. Akad. Nauk 368 (1999), no. 6, 771-773; English transl., Dokl. Phys. 44 (1999), no. 10, 715-717. MR $1749046(2001 \mathrm{c}: 74043)$

[8] G. Duvaut and J.-L. Lions, Les inéquations en mécanique et en physique, Travaux Rech. Math., No. 21, Dunod, Paris, 1972. MR0464857 (57:4778)

[9] C. Amrouche and V. Girault, Decomposition of vector spaces and application to the Stokes problem in arbitrary dimension, Czechoslovak Math. J. 44 (1994), 109-140. MR1257940 (95c:35190)

[10] I. M. Glazman, Direct methods of qualitative spectral analysis of singular differential operators, Fizmatgiz, Moscow, 1963; English transl., Daniel Davey and Co., Inc., New York, 1966. MR0185471 (32:2938) MR0190800 (32:8210)

[11] M. Sh. Birman and M. Z. Solomyak, Spectral theory of selfadjoint operators in Hilbert space, Leningrad. Univ., Leningrad, 1980; English transl., Reidel, Dordrecht, 1987. MR0609148 (82k:47001) MR:1192782(93g:47001)

E-mail address: i.kamotski@maths.bath.ac.uk

Received 5/APR/2007

Translated by A. PLOTKIN 